\title{
SHARPENDING OF THE VNIR AND SWIR BANDS OF THE WIDE BAND SPECTRAL IMAGER ONBOARD TIANGONG-II IMAGERY USING THE SELECTED BANDS
}

\author{
Qingsheng Liu ${ }^{1} *$, Xinyang $\mathrm{Li}^{1}$, Gaohuan Liu ${ }^{1}$, Chong Huang ${ }^{1}, \mathrm{He} \mathrm{Li}^{1}$, Xudong Guan ${ }^{1}$ \\ ${ }^{1}$ State Key Laboratory of Resources and Environmental Information System, Institute of Geographic Sciences and Natural \\ Resources Research, CAS, Beijing, China - (liuqs, lixy, liugh, huangch, lih, guanxd)@lreis.ac.cn
}

Commission VI, WG III/6

KEY WORDS: Sharpening, band selection, Tiangong-II, VNIR and SWIR bands, Wide band spectral imager

\begin{abstract}
:
The Tiangong-II space lab was launched at the Jiuquan Satellite Launch Center of China on September 15, 2016. The Wide Band Spectral Imager (WBSI) onboard the Tiangong-II has 14 visible and near-infrared (VNIR) spectral bands covering the range from 403-990 nm and two shortwave infrared (SWIR) bands covering the range from 1230-1250 nm and 1628-1652 nm respectively. In this paper the selected bands are proposed which aims at considering the closest spectral similarities between the VNIR with $100 \mathrm{~m}$ spatial resolution and SWIR bands with $200 \mathrm{~m}$ spatial resolution. The evaluation of Gram-Schmidt transform (GS) sharpening techniques embedded in ENVI software is presented based on four types of the different low resolution pan band. The experimental results indicated that the VNIR band with higher CC value with the raw SWIR Band was selected, more texture information was injected the corresponding sharpened SWIR band image, and at that time another sharpened SWIR band image preserve the similar spectral and texture characteristics to the raw SWIR band image.
\end{abstract}

\section{INTRODUCTION}

The Wide Band Spectral Imager (WBSI) onboard the TiangongII space lab was launched at the Jiuquan Satellite Launch Center of China on September 15, 2016 which has 14 visible and nearinfrared (VNIR) spectral bands covering the range from 403$990 \mathrm{~nm}$ at a spatial resolution of $100 \mathrm{~m}$ and two shortwave infrared (SWIR) bands with $200 \mathrm{~m}$ spatial resolution covering the range from 1230-1250 $\mathrm{nm}$ and 1628-1652 nm respectively. The spectral range of each band is shown in Table 1. The WBSI images the Earth's surface in 300-killometer-wide swath which supports a wide range of applications such areas as plant, agriculture, forestry, geology, geography, ocean, water resource, natural hazard, and coastal researches.

\begin{tabular}{|l|c|c|}
\hline Bands & Wavelength & Resolution \\
\hline VNIR Band 1 & nm & $\mathrm{m}$ \\
VNIR Band 2 & $970-990$ & 100 \\
VNIR Band 3 & $830-950$ & 100 \\
VNIR Band 4 & $845-915$ & 100 \\
VNIR Band 5 & $810-830$ & 100 \\
VNIR Band 6 & $740-760$ & 100 \\
VNIR Band 7 & $677.5-687.5$ & 100 \\
VNIR Band 8 & $655-675$ & 100 \\
VNIR Band 9 & $610-630$ & 100 \\
VNIR Band 10 & $555-575$ & 100 \\
VNIR Band 11 & $510-530$ & 100 \\
VNIR Band 12 & $480-500$ & 100 \\
VNIR Band 13 & $433-453$ & 100 \\
VNIR Band 14 & $403-423$ & 100 \\
SWIR Band 1 & $1230-1250$ & 100 \\
SWIR Band 2 & $1628-1652$ & 200 \\
\hline
\end{tabular}

Table 1 . The wavelength of each band of Tiangong-II
The SWIR bands are out of the visible light range. The SWIR bands can detect the minerals, man-made materials, plant water status, soil moisture and fire, and see in night and through smoke in a way that is invisible to the VNIR bands (Matheson and Dennison, 2012; Sanchez-Ruiz et al., 2014; Young, 2015). Therefore, it is often hopeful to have a high spectral resolution and spatial information combined in the same file (Padwick et al., 2010). The integration of the WBSI imagery of Tiangong-II is difficult because the VNIR and SWIR bands have the different spatial resolutions. Except the images are resized by the nearest neighbour or cubic convolution interpolation methods so that all VNIR and SWIR bands have the same size of pixels (Wahi et al., 2013; Pour and Hashim, 2013), thus sharpening should be used to combine the low resolution SWIR image with the high resolution VNIR image to produce a spatial resolution of $100 \mathrm{~m}$ image including 14 VNIR bands and two SWIR bands.

Many sharpening techniques exist in the literature (Vaiopoulos and Karantzalos, 2016). Some of them have been embedded in ENVI software, the famous commercial remote sensing software, and thus make the image analyzers convenient to automatically fusion the different spectral and spatial resolution images. However, the above methods cannot be directly used because the WBSI onboard Tiangong-II has not a panchromatic band. Moreover, the similarity between the low spatial resolution images and high spatial resolution images is crucial for the quality of the fusion images (Aiazzi et al., 2005; Nikolakopoulos, 2006; Pak etl al., 2017). But the VNIR bands of the WBSI do not overlap spectrally with the SWIR bands. To sharpen a WBSI image of Tiangong-II, a specific multispectral band should be considered as an optimal panchromatic band with $100 \mathrm{~m}$ spatial resolution. The selected and synthesized bands from the existed multispectral bands is an effective

* Corresponding author 
approach, which has been successfully applied in sharpening the VNIR and SWIR bands of Sentinel-2 and the VNIR, SWIR and thermal infrared bands of ASTER (Aiazzi et al., 2005; Du et al., 2016; Vaiopoulos and Karantzalos, 2016; Wang et al., 2016; Park et al., 2017).

The main goal of this work was to sharpen the two SWIR bands of the WBSI imagery of Tiangong-II with $200 \mathrm{~m}$ spatial resolution using the selected higher spatial resolution VNIR bands at $100 \mathrm{~m}$ and evaluate Gram-Schmidt transform (GS) embedded in ENVI software for sharpening of the SWIR bands of the WBSI.

\section{MATERIALS AND METHODS}

\subsection{Data and Experiment Area}

The WBSI Level 2 data was acquired on March 18, 2017, which was geometrically corrected product and covered the small areas of southern Tianjin City and the southeast of Hebei Province, and large areas of the northwest of Shandong Province. Three sub-regions were selected for the experiment area covered by $400 \times 400$ pixels in bands with $100 \mathrm{~m}$ spatial resolution as shown in Figure 1 . The main reasons for the selection were, (1) to contain a variety of land cover classes including crop, forest, bare soil, resident, aquaculture, river, Sea and so on. The left is Area 1 where mainly had high-winterwheat-yield land and resident. The middle is Area 2 where mainly had the middle and low-winter-wheat-yield land, unsown land, resident, small reservoirs and winter jujube. The right is Area 3 where mainly had the middle-winter-wheat-yield land, resident, river, Sea, aquaculture, unsown land and forest; (2) exclude the lower right areas including cloud; (3) these region are also the core demonstration zones of Chinese $\mathrm{R} \& \mathrm{D}$ Demonstration Project on Bohai-rim's Breadbasket which aims to increase crop production by 3 billion $\mathrm{kg}$ by 2017 and 5 billion by 2020 .

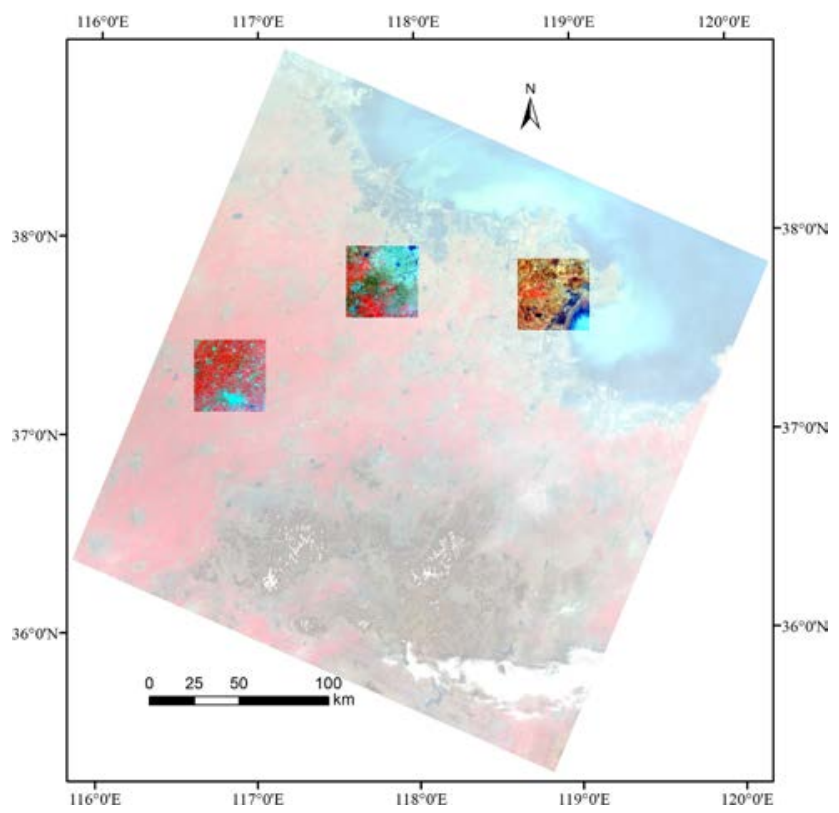

Figure 1. The relative location and size of the WBSI image of the Tiangong-II of the three experiment areas $(400 \times 400$ pixels

in bands with $100 \mathrm{~m}$ spatial resolution, the left, Area 1; the middle, Area 2; the right, Area 3). A false colour composite is displayed (RGB B5-B8-B10)

\subsection{Sharpening Method Used in This Work}

In ENVI software version 5.1, two image sharpening methods for byte-scaled RGB imagery include HSV transform (HSV) and Colour normalization transform (Brovey) and three image sharpening techniques for spectral imagery include GramSchmidt transform (GS), Principal components transform (PC), and Colour normalized transform (CN). The descriptions on the algorithms of the aforementioned sharpening methods can be found in ENVI software version 5.1 help documents and some references (Vrabel, 1996; Vrabel et al., 2002; Nikolakopoulos, 2005; Sarp, 2014). GS was one of the sharpening techniques with preserving the spectral characteristics and minimum information loss (Sarp, 2014; Gao et al., 2016). In this work, GS sharpening method was applied, and the sharpening results from three sub-regions were evaluated. In this study, the GS sharpening procedure was the same every time except choosing the different low resolution pan band such as the SWIR Band 1, the SWIR Band2, average of the SWIR Band 1 and SWIR Band 2, and the high resolution VNIR Band.

\subsection{Assessments of the Sharpening Images}

Images are prone to spectral distortions during sharpening operations, especially if a high spatial resolution image not overlapped spectrally by the low spatial resolution multispectral bands or does not have similar spectral characteristics (Sarp, 2014; Pak et al., 2017). In this work, the statistical parameters of the histogram, the minimum, the maximum, the mean, the standard deviation (St. dev.) value and correlation coefficient (CC) were used in order to select the optimal VNIR band as the panchromatic band and evaluate the quality of the sharpening images (Nikolakopoulos, 2006; Sarp, 2014).

\section{RESULTS AND DISCUSSIONS}

\subsection{Selected Band Analysis}

The CC is used to statistically measure the intensity of a linear association between two images. In this work, the CC was applied to determine the selected bands from the set of VNIR bands which had the high correlations with each SWIR bands. Firstly, the VNIR bands were resized to match the spatial resolution of the SWIR bands using the nearest neighbour algorithm which uses the nearest pixel values without interpolation to preserve the original pixel values of the SWIR bands. The opposite process was also done to more fully understand the correlations between the VNIR and SWIR bands.

Table 1, Table 2, Table 3, Table 4, Table 5 and Table 6 described the CC between the VNIR and SWIR bands. The more similarity between the VNIR bands and the SWIR bands showed the higher correlation coefficient values. It showed that the CC values of Area 3 were higher than those of Area 2, and those of Area 2 were higher than those of Area 1, which indicated that the more water areas the higher correlation coefficient values between the VNIR and SWIR bands. Compared Table 1 with Table 4, Table 2 with Table 3, and Table 3 and Table 6, the CC values between the VNIR and SWIR bands with $100 \mathrm{~m}$ spatial resolution were higher than those with $200 \mathrm{~m}$ spatial resolution at the Area 1, whereas the CC values between the SWIR Band 1and the SWIR Band 2 was lower. But at the Area 2 and Area 3, the opposite results were gotten. It showed that the CC values between the VNIR and SWIR bands with the downscaled results were higher than those with the upscaled results, and the CC values between the SWIR 
Band 1 and Band 2 with the downscaled results were slightly lower than those with the upscaled results, which indirectly reflected the sensitivities of the SWIR Band 1 and Band2 to water areas.

\begin{tabular}{|l|c|c|c|c|}
\hline Bands & SWIR 1 & SWIR 2 & Mean & St. dev. \\
\hline VNIR 1 & 0.8660 & -0.3934 & 621.0 & 47.8 \\
VNIR 2 & 0.8100 & -0.3897 & 283.1 & 19.3 \\
VNIR 3 & 0.8409 & -0.4482 & 638.3 & 55.3 \\
VNIR 4 & 0.8497 & -0.4701 & 943.7 & 85.0 \\
VNIR 5 & 0.8482 & -0.4793 & 866.6 & 71.5 \\
VNIR 6 & 0.8630 & -0.4584 & 1143.3 & 75.5 \\
VNIR 7 & -0.5923 & 0.6759 & 829.8 & 55.1 \\
VNIR 8 & -0.59992 & 0.6734 & 881.1 & 55.1 \\
VNIR 9 & -0.5966 & 0.6560 & 945.6 & 45.3 \\
VNIR 10 & -0.5481 & 0.5962 & 1128.7 & 34.6 \\
VNIR 11 & -0.5538 & 0.5510 & 1296.2 & 34.5 \\
VNIR 12 & -0.5628 & 0.5251 & 1496.5 & 37.0 \\
VNIR 13 & -0.5145 & 0.4600 & 1006.4 & 19.7 \\
VNIR 14 & -0.4772 & 0.4104 & 1715.8 & 28.7 \\
SWIR 1 & 1.0000 & -0.2216 & 150.7 & 7.4 \\
SWIR 2 & -0.2216 & 1.0000 & 66.4 & 4.5 \\
\hline
\end{tabular}

Table 1. The CC, Mean and St. dev. for the VNIR and SWIR bands with $100 \mathrm{~m}$ spatial resolution of the Area 1

\begin{tabular}{|l|c|c|c|c|}
\hline Bands & SWIR 1 & SWIR 2 & Mean & St. dev. \\
\hline VNIR 1 & 0.8674 & 0.3822 & 558.5 & 47.4 \\
VNIR 2 & 0.7388 & 0.1751 & 236.4 & 19.2 \\
VNIR 3 & 0.8044 & 0.2361 & 561.9 & 51.1 \\
VNIR 4 & 0.8160 & 0.2511 & 870.3 & 77.9 \\
VNIR 5 & 0.7929 & 0.2097 & 808.7 & 66.9 \\
VNIR 6 & 0.8027 & 0.2443 & 1056.6 & 69.9 \\
VNIR 7 & 0.1365 & 0.7136 & 1078.4 & 63.8 \\
VNIR 8 & 0.1195 & 0.6992 & 1130.4 & 62.8 \\
VNIR 9 & 0.0526 & 0.6239 & 1167.5 & 51.5 \\
VNIR 10 & -0.0255 & 0.4855 & 1310.2 & 39.8 \\
VNIR 11 & -0.0100 & 0.5006 & 1478.8 & 36.9 \\
VNIR 12 & -0.0202 & 0.4992 & 1676.0 & 36.8 \\
VNIR 13 & 0.0037 & 0.4346 & 1078.8 & 16.7 \\
VNIR 14 & 0.0247 & 0.3363 & 1803.6 & 22.3 \\
SWIR 1 & 1.0000 & 0.6817 & 140.8 & 10.6 \\
SWIR 2 & 0.6817 & 1.0000 & 78.5 & 7.7 \\
\hline
\end{tabular}

Table 2. The CC, Mean and St. dev. for the VNIR and SWIR bands with $100 \mathrm{~m}$ spatial resolution of the Area 2

\begin{tabular}{|l|c|c|c|c|}
\hline Bands & SWIR 1 & SWIR 2 & Mean & St. dev. \\
\hline VNIR 1 & 0.9565 & 0.9447 & 507.3 & 99.4 \\
VNIR 2 & 0.9424 & 0.9286 & 218.2 & 30.2 \\
VNIR 3 & 0.9323 & 0.9147 & 508.6 & 83.3 \\
VNIR 4 & 0.9224 & 0.9031 & 796.1 & 124.8 \\
VNIR 5 & 0.8766 & 0.8569 & 748.7 & 91.2 \\
VNIR 6 & 0.8685 & 0.8499 & 1035.5 & 103.8 \\
VNIR 7 & 0.3175 & 0.3678 & 1198.3 & 66.8 \\
VNIR 8 & 0.2194 & 0.2718 & 1254.0 & 65.0 \\
VNIR 9 & -0.1923 & -0.1407 & 1297.7 & 56.4 \\
VNIR 10 & -0.5058 & -0.4635 & 1445.7 & 54.8 \\
VNIR 11 & -0.4654 & -0.4201 & 1611.2 & 44.3 \\
VNIR 12 & -0.4949 & -0.4482 & 1808.7 & 43.0 \\
VNIR 13 & -0.6027 & -0.5586 & 1141.7 & 21.6 \\
VNIR 14 & -0.6647 & -0.6234 & 1875.3 & 32.7 \\
SWIR 1 & 1.0000 & 0.9880 & 126.2 & 31.4 \\
SWIR 2 & 0.9880 & 1.0000 & 71.1 & 21.0 \\
\hline
\end{tabular}

Table 3. The CC, Mean and St. dev. for the VNIR and SWIR bands with $100 \mathrm{~m}$ spatial resolution of the Area 3

\begin{tabular}{|l|c|c|c|c|}
\hline Bands & SWIR 1 & SWIR 2 & Mean & St. dev. \\
\hline VNIR 1 & 0.7097 & -0.2562 & 626.9 & 42.2 \\
VNIR 2 & 0.6723 & -0.2634 & 282.9 & 17.3 \\
VNIR 3 & 0.6898 & -0.2967 & 646.3 & 49.7 \\
VNIR 4 & 0.7000 & -0.3129 & 963.0 & 75.9 \\
VNIR 5 & 0.7021 & -0.3212 & 882.1 & 63.9 \\
VNIR 6 & 0.7247 & -0.3119 & 1159.1 & 65.8 \\
VNIR 7 & -0.4671 & 0.4671 & 819.6 & 48.8 \\
VNIR 8 & -0.4272 & 0.4732 & 869.6 & 48.2 \\
VNIR 9 & -0.4138 & 0.4836 & 933.9 & 38.1 \\
VNIR 10 & -0.3382 & 0.4863 & 1117.0 & 26.7 \\
VNIR 11 & -0.3323 & 0.4755 & 1283.0 & 24.6 \\
VNIR 12 & -0.3467 & 0.4655 & 1480.4 & 25.2 \\
VNIR 13 & -0.2908 & 0.4136 & 995.8 & 12.9 \\
VNIR 14 & -0.2434 & 0.3600 & 1699.1 & 18.9 \\
SWIR 1 & 1.0000 & -0.2419 & 152.4 & 5.8 \\
SWIR 2 & -0.2419 & 1.0000 & 65.7 & 4.3 \\
\hline
\end{tabular}

Table 4. The CC, Mean and St. dev. for the VNIR and SWIR bands with $200 \mathrm{~m}$ spatial resolution of the Area 1

\begin{tabular}{|l|c|c|c|c|}
\hline Bands & SWIR 1 & SWIR 2 & Mean & St. dev. \\
\hline VNIR 1 & 0.8823 & 0.3921 & 558.6 & 47.5 \\
VNIR 2 & 0.7504 & 0.1812 & 236.5 & 19.3 \\
VNIR 3 & 0.8174 & 0.2430 & 562.1 & 51.2 \\
VNIR 4 & 0.8290 & 0.2581 & 870.6 & 78.1 \\
VNIR 5 & 0.8051 & 0.2159 & 809.0 & 67.1 \\
VNIR 6 & 0.8155 & 0.2515 & 1056.8 & 70.1 \\
VNIR 7 & 0.1386 & 0.7264 & 1077.2 & 63.9 \\
VNIR 8 & 0.1214 & 0.7117 & 1129.2 & 62.9 \\
VNIR 9 & 0.0535 & 0.6342 & 1166.4 & 51.6 \\
VNIR 10 & -0.0249 & 0.4937 & 1309.3 & 39.9 \\
VNIR 11 & -0.0087 & 0.5088 & 1477.9 & 37.0 \\
VNIR 12 & -0.0189 & 0.5072 & 1675.1 & 37.0 \\
VNIR 13 & 0.0055 & 0.4427 & 1078.4 & 16.8 \\
VNIR 14 & 0.0265 & 0.3443 & 1803.0 & 22.4 \\
SWIR 1 & 1.0000 & 0.6779 & 140.8 & 10.7 \\
SWIR 2 & 0.6779 & 1.0000 & 78.4 & 7.7 \\
\hline
\end{tabular}

Table 5. The CC, Mean and St. dev. for the VNIR and SWIR bands with $200 \mathrm{~m}$ spatial resolution of the Area 2

\begin{tabular}{|l|c|c|c|c|}
\hline Bands & SWIR 1 & SWIR 2 & Mean & St. dev. \\
\hline VNIR 1 & 0.9618 & 0.9508 & 508.3 & 98.9 \\
VNIR 2 & 0.9475 & 0.9344 & 218.6 & 30.0 \\
VNIR 3 & 0.9373 & 0.9203 & 509.0 & 83.0 \\
VNIR 4 & 0.9273 & 0.9086 & 797.4 & 124.3 \\
VNIR 5 & 0.8817 & 0.8625 & 749.6 & 90.9 \\
VNIR 6 & 0.8736 & 0.8555 & 1036.2 & 103.5 \\
VNIR 7 & 0.3229 & 0.3763 & 1198.0 & 66.5 \\
VNIR 8 & 0.2246 & 0.2800 & 1253.6 & 64.7 \\
VNIR 9 & -0.1880 & -0.1342 & 1297.1 & 56.2 \\
VNIR 10 & -0.5045 & -0.4589 & 1445.0 & 54.6 \\
VNIR 11 & -0.4626 & -0.4158 & 1610.5 & 44.2 \\
VNIR 12 & -0.4926 & -0.4445 & 1808.0 & 43.0 \\
VNIR 13 & -0.6007 & -0.5556 & 1141.4 & 21.6 \\
VNIR 14 & -0.6623 & -0.6205 & 1875.0 & 32.8 \\
SWIR 1 & 1.0000 & 0.9879 & 126.4 & 31.3 \\
SWIR 2 & 0.9879 & 1.0000 & 71.2 & 20.9 \\
\hline
\end{tabular}

Table 6. The CC, Mean and St. dev. for the VNIR and SWIR bands with $200 \mathrm{~m}$ spatial resolution of the Area 3 
For the areas with more water bodies such as Area 3, all of the CC values between the VNIR Band 1-6 and the SWIR Band 1 and Band 2 were higher than 0.85. The maximum value in CC was observed at the VNIR Band1, whereas the maximum value in Mean and St. dev. of these six VNIR band was observed at the VNIR Band 6. For the areas with a few water bodies such as Area 2 and Area 1, the CC values between the VNIR Band 1-6 and the SWIR Band 1 were higher than 0.67. The maximum value in CC was also observed at the VNIR Band 1, whereas the maximum value in Mean and St. dev. of these six VNIR band was observed at the VNIR Band 6. Thus, it needs to evaluate these six VNIR bands to select the optimal band for sharpening the SWIR Band 1.

In Area 1 and Area2, for the SWIR Band 2, the CC values between the VNIR Band 7-9 and the SWIR Band 2 were higher than 0.60 . Except the Area 1 with the downscaled $200 \mathrm{~m}$ spatial resolution image (Table 4), the maximum value in CC occurred at the VNIR Band 7, whereas the maximum value in Mean and St. dev. of these six VNIR band was observed at the VNIR Band 9 and Band 7 respectively. Thus, it needs to evaluate these three VNIR bands to select the optimal band for sharpening the SWIR Band 2.

It is noteworthy that the CC values between the VNIR bands and the SWIR Band 2 for Area 1 with the downscaled $200 \mathrm{~m}$ spatial resolution image were less than 0.50 and the maximum value was observed at the VNIR Band 10. But Area 1 was the core demonstration zones for increasing winter wheat production. Therefore, it needs to evaluate the VNIR Band 7-12 to select the optimal band for sharpening the SWIR Band 2.

\subsection{Comparison of the Sharpening Images}

Quantitative and qualitative assessments could be performed on comparison of the sharpening images. A qualitative comparison was done through visual inspection of gray level and texture changes (Figure 2-4). Quantitative evaluations could be done through consistency measurements between the sharpened images and the available reference images (Nikolakopoulos, 2006). When no reference image existed, consistency measurements were known to be efficient at estimating a sharpened image using the correction coefficient (CC), root mean square error (RMSE), structural similarity index (SSIM), the erreur relative global adimensionnelle de synthese (ERGAS), universal image quality index (UIQI) and so on (Sarp, 2014; Vaiopoulos and Karantzalos, 2016; Pak et al, 2017). Nevertheless, we did not agree that it was a good idea to evaluate the sharpened images through consistency measurements between the sharpened images and the downscaled low spatial resolution images. Therefore, a quantitative evaluation was performed to examine the spectral information preservation through the eight statistical parameters of the histogram, the minimum, the maximum, the mean, the St. dev., the median, the mode, the skewness and the kurtosis (Figure 5-7).

Figure 5, Figure 6 and Figure 7 showed that the mean and median of the sharpened images from the different VNIR band as the high resolution pan band and four different selections as the low resolution pan band rarely deviated from those of the raw $200 \mathrm{~m}$ SWIR band images for the Area 1, Area 2 and Area 3 , indicating the whole radiance levels were stable for the before and after sharpening images. The sharpened images and the raw $200 \mathrm{~m}$ SWIR band image had the similar mode values at the Area 1 and Area 2. For the Area 3, most of the mode values of the sharpened images were deviated from that of the raw SWIR band images except the VNIR Band 1.

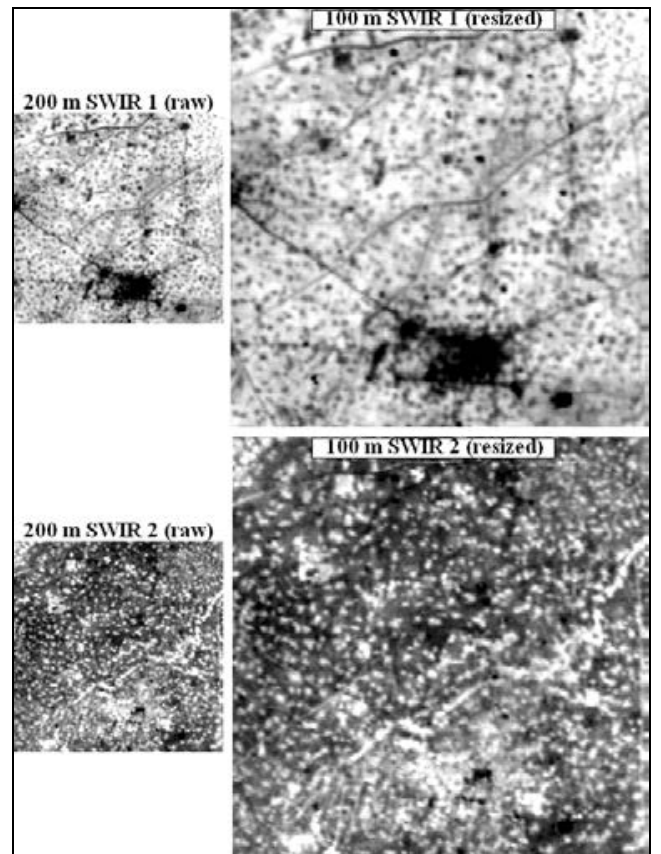

Figure 2. The raw and resized images on the SWIR bands of the WBSI of Tiangong-II at the Area 1

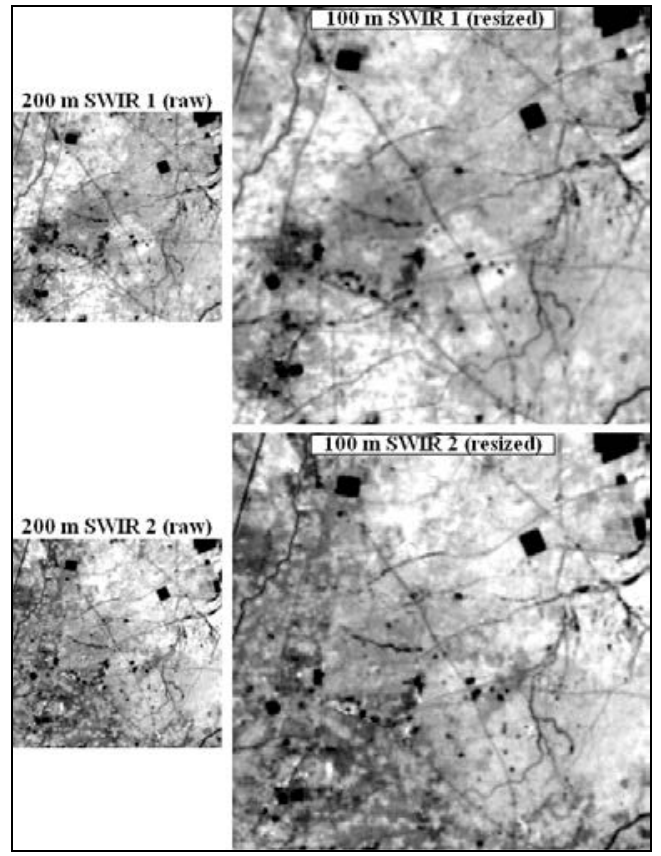

Figure 3. The raw and resized images on the SWIR bands of the WBSI of Tiangong-II at the Area 2

The differences of the maximum values between the sharpened images from the different high resolution VNIR bands using four types of low resolution pan bands and the raw SWIR bands were relatively small, whereas the differences of the minimum values were relatively big (just the same as the statement by Nikolakopoulos (2006)), especially when the downscaled VNIR bands selected as the low resolution pan bands during the GS sharpening procedure. The skewness and St. dev. values of the sharpened images deviated from the raw SWIR band images 
seemed to be less than that of the kurtosis. It noted that from Area 1 to Area 3 with the increasing water areas the deviation of the skewness, the kurtosis and St. dev. values of the sharpened images from those of the raw SWIR band images gradually decreased.

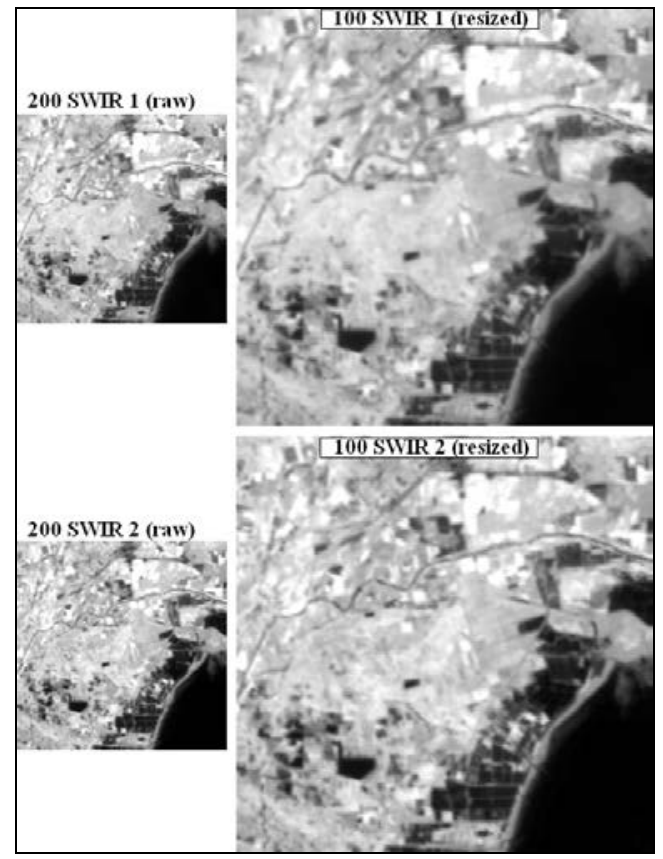

Figure 4. The raw and resized images on the SWIR bands of the WBSI of Tiangong-II at the Area 3

In general, when the SWIR Band 1 was selected as the low resolution pan band, the mean and St. dev. of the sharpened images were almost identical to that of the raw $200 \mathrm{~m}$ SWIR Band 1 image, and when the SWIR Band 2 was selected as the low resolution pan band, the mean and St. dev. of the sharpened images were almost identical to that of the raw $200 \mathrm{~m}$ SWIR Band 2 image. For four types of the low resolution pan bands, the minimum, maximum, mean, St. dev. values of sharpened images from the first six VNIR bands using the VNIR band as the low resolution pan band were far from those of the raw 200 $m$ SWIR bands, whereas those of the VNIR Band 7-Band 12 were similar to those of the raw $200 \mathrm{~m}$ SWIR bands, indicating the relationships between the VNIR band and the SWIR band reduced the consistency of the sharpened image and the raw SWIR band when choosing the downscaled VNIR band as the low resolution pan band. At the Area 1, the kurtosis values of the sharpened SWIR Band 1 image with the SWIR Band 2 used as the low resolution pan band were greater than those from the SWIR Band 1 used as the low resolution pan band, moreover, the higher the CC were the greater the kurtosis values were, and vice versa. When the average of the SWIR bands were used as the low resolution pan band, the kurtosis and skewness values of the sharpened SWIR Band 1images from the six VNIR bands were smallest of four types of the low resolution pan band at the Area 1 and Area 2, indicating the trend to normal distribution of spectral values.

The higher the CC values between the VNIR bands with the SWIR Band 1 were more similar the skewness values of the sharpened SWIR Band 2 images were with the raw $200 \mathrm{~m}$ SWIR Band 2, and for the Area 3 it was also true for the kurtosis values. For the image with more water areas such as the
Area 3 the statistical characteristics of the sharpened image with the downscaled VNIR bands as the low resolution pan band was obviously deviated from those of the raw SWIR bands although the spectral range was stretched which may be helpful for image classification. The sharpened SWIR Band 1 image from the VNIR Band 1 and the SWIR Band 1 selected as the high resolution pan and the low resolution pan band respectively were more similar to the raw $200 \mathrm{~m}$ SWIR bands, which indicated the VNIR Band 1 may be the optimal sharpening band for the SWIR for the Area 3 and similar images.
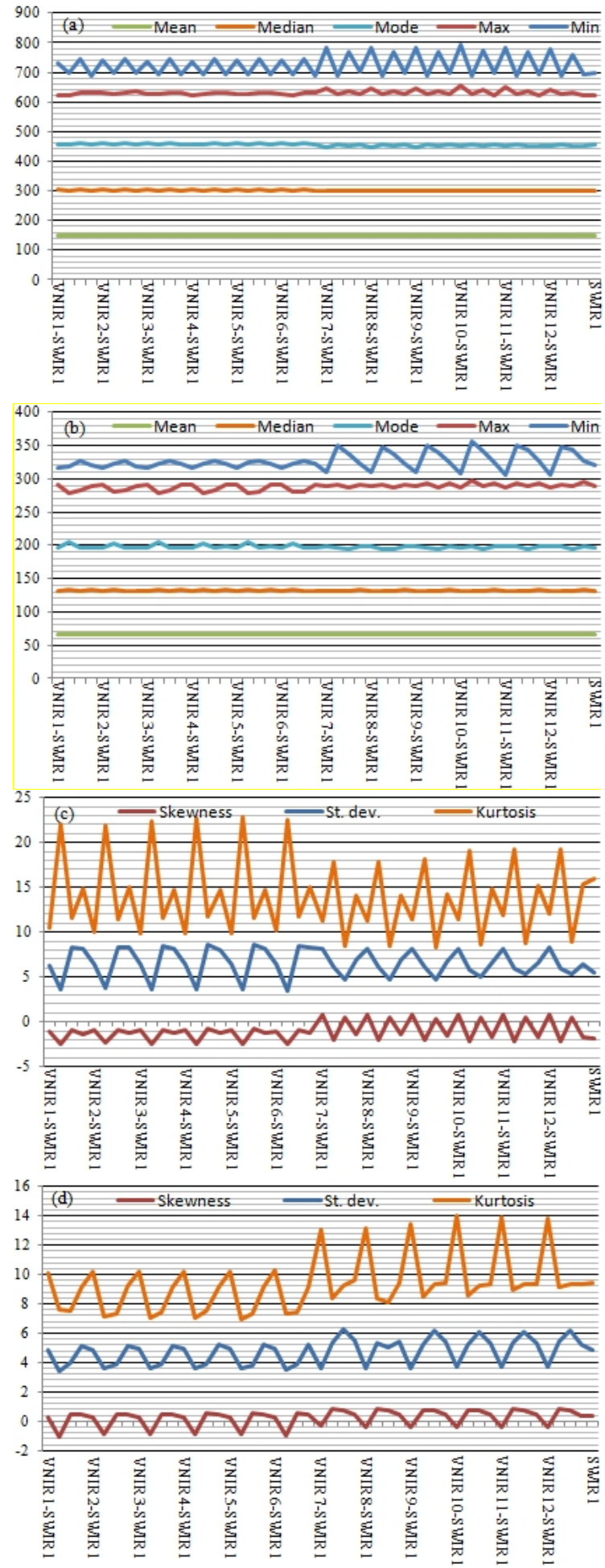
Figure 5. The minimum, maximum, mean, St. dev., median, mode, skewness and kurtosis of the sharpened images using the four types of low resolution pan band and raw images on the SWIR bands of the WBSI of Tiangong-II at the Area 1
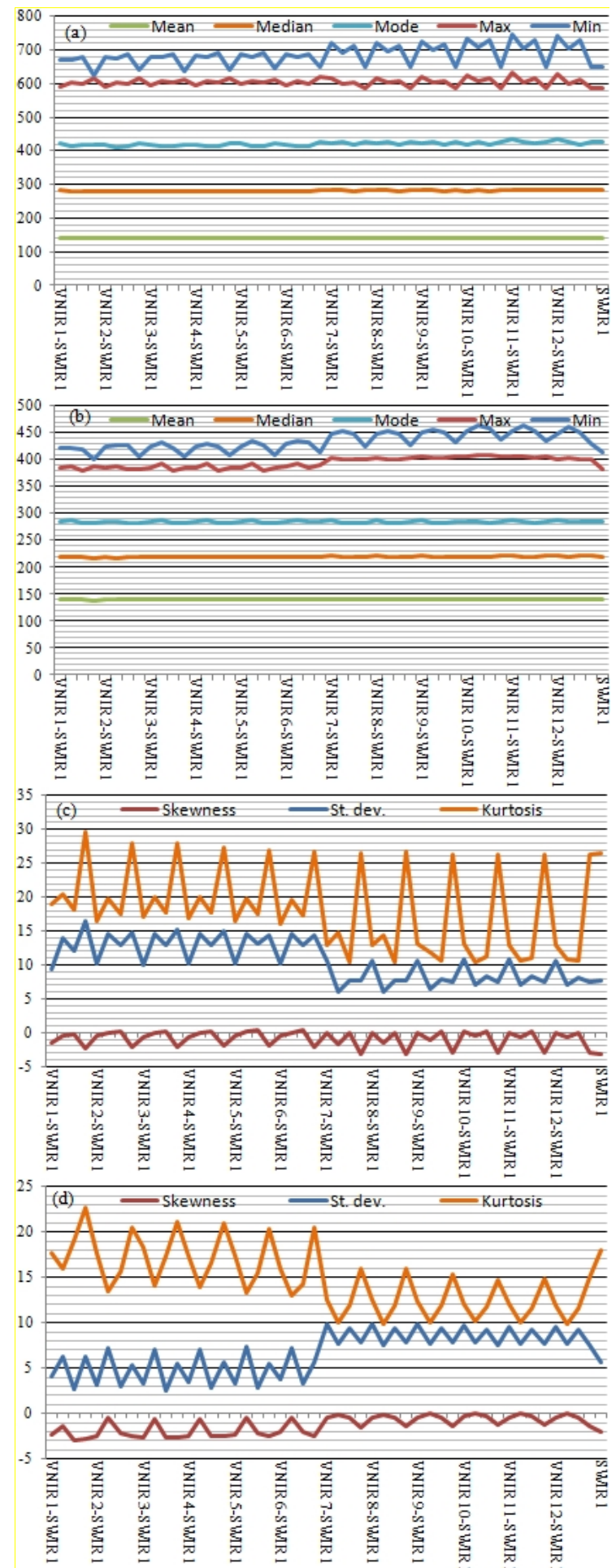

Figure 6. The minimum, maximum, mean, St. dev., median, mode, skewness and kurtosis of the sharpened images using the four types of low resolution pan band and raw images on the SWIR bands of the WBSI of Tiangong-II at the Area 2
For the Area 2, the optimal sharpened SWIR Band 1 image which had the similar spectral characteristics with the raw SWIR Band 1and enhanced texture information was got when the VNIR Band 1 was used as the high resolution pan band with the raw $200 \mathrm{~m}$ SWIR Band 1 was used as the low resolution pan band, and the optimal sharpened SWIR Band 2 image which had the similar spectral characteristics with the raw SWIR Band 1and enhanced texture information was got when the VNIR Band 7 was used as the high resolution pan band and the downscaled $200 \mathrm{~m}$ VNIR Band 1 was used as the low resolution pan band. For the Area 3, the difference between the SWIR Band 1 and SWIR Band 2 was small (Figure 4), moreover, the CC values between the SWIR bands and the first six VNIR bands were greater than 0.85 (Table 6), it was difficult to evaluate the sharpened image by visual inspection, as mentioned above, the VNIR Band 1 may be the optimal sharpening band for the SWIR for the Area 3 and similar images.

For the image with fewer water areas and more vegetation such as the Area 1, compared with the SWIR Band 1 or the SWIR Band 2 used as the low resolution pan band, only one of two sharpened SWIR band images had the enhanced spatial and texture information, all of the sharpened two SWIR band images from the first six VNIR bands used as the high resolution pan band and the average of two SWIR bands or the downscaled VNIR band selected as the low resolution pan got the spatial and texture information enhancement. From the similarity of visual inspection, the later was better (Figure 8). Moreover, when the downscaled VNIR band was used as the low resolution pan band, the sharpened SWIR band images from the fourteen VNIR bands were similar, in other words, the CC values between the VNIR bands and the SWIR bands had few effects on the sharpen images. Of course, the higher the CC values were, the higher the quality of the sharpen images were. However, it was noteworthy that it was susceptible to the registration between the downscaled VNIR bands and the SWIR bands. When the VNIR band with the higher negative CC values with the SWIR Band 1 such as the VINR Band 7-14 at the Area 1, the sharpened SWIR Band 1 image with the SWIR Band 1 used as the low resolution pan band and the sharpened SWIR Band 2 image with the SWIR Band 2 used as the low resolution pan band and all of two sharpened SWIR bands with the average of two SWIR bands used as the low resolution pan band had the inverse spectral characteristics to the raw $200 \mathrm{~m}$ SWIR bands. For the first six VNIR bands, by visual inspection, the sharpened SWIR Band 1 images using the same VNIR band as the low resolution pan band was most similar to the raw SWIR Band 1 image, and the second was using the SWIR Band 2 as the low resolution pan band but had the poor image quality, and that of the SWIR Band 1 or the average of two SWIR bands as the low resolution pan band had more detailed texture information, especially that of the SWIR Band 1 used as the low resolution pan band was similar to the VNIR band. Except the SWIR Band 2 used as the low resolution pan band, the sharpened SWIR Band 2 images from the other three types of the row resolution pan band had the similar spectral and texture characteristics to the raw SWIR Band 2 image, that of the average of two SWIR bands as the low resolution pan band had more detailed texture information but had more noises. When the CC values between the VNIR bands and the SWIR Band 2 increased, the sharpened SWIR Band 2 image from the SWIR Band 1 used as the low resolution pan band and the sharpened SWIR Band 1 image from the SWIR Band 2 used as the low resolution pan band was more similar to the corresponding raw SWIR band images. 


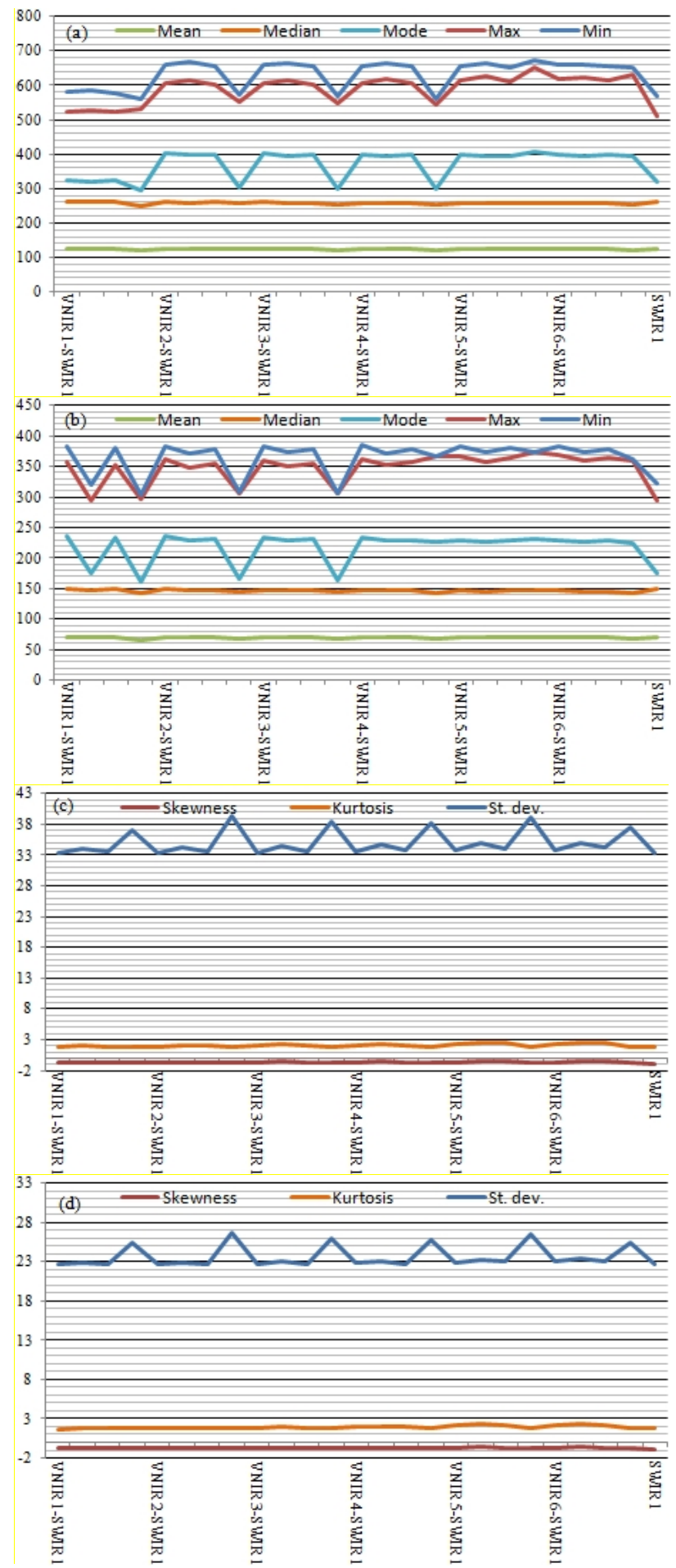

Figure 7. The minimum, maximum, mean, St. dev., median, mode, skewness and kurtosis of the sharpened images using the four types of low resolution pan band and raw images on the SWIR bands of the WBSI of Tiangong-II at the Area 3

When the VNIR band with the high positive CC value with the raw SWIR Band 1 was used to sharpen the SWIR image, more texture information was injected the sharpened SWIR Band 1 image, and the sharpened SWIR Band 2 image had more similar to the raw SWIR Band 2 image and vice versa. When the VNIR band with the relatively high negative $\mathrm{CC}$ value with the raw SWIR Band 1 and the relatively high positive CC value with the raw SWIR Band 2 was used to sharpen the SWIR image, more texture information was injected the sharpened SWIR Band 1 image but with the inverse spectral characteristics to the raw SWIR Band 1 image, and the sharpened SWIR Band 2 image had more similar to the raw SWIR Band 2 image and vice versa.
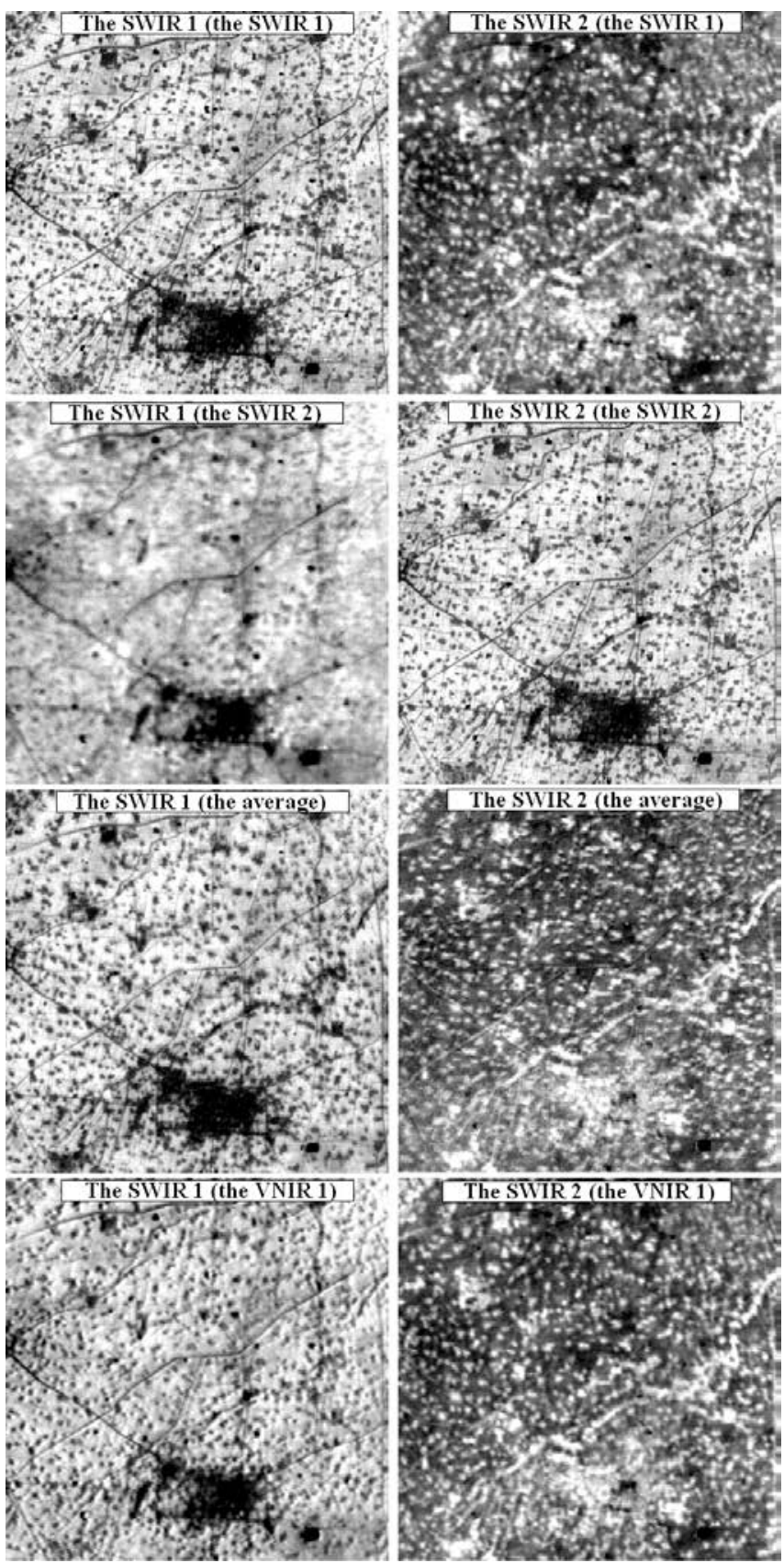

Figure 8. The sharpened SWIR image for the Area 1

\section{CONCLUSSIONS}

Although the $200 \mathrm{~m}$ resolution SWIR images from the WBSI of Tiangong-II can be simply resized by the nearest neighbour or cubic convolution interpolation methods to correspond to the VNIR $100 \mathrm{~m}$ spatial resolution images. But as shown in Figure 2 to Figure 4, the resized image definition was not so desirable. Therefore, sharpening should be used to combine the low resolution SWIR image with the high resolution VNIR image to produce a spatial resolution of $100 \mathrm{~m}$ image including 14 VNIR bands and two SWIR bands. In this work, GS sharpening 
method embedded in ENVI software was applied based on four types of the different low resolution pan band such as the SWIR Band 1, the SWIR Band2, the average of the SWIR Band 1 and SWIR Band 2, and the high resolution VNIR Band. For the image with more water areas such as the Area 3, the VNIR Band 1 may be the optimal sharpening band. For the Area 2 with a few water and vegetated areas, the optimal sharpened SWIR Band 1 image was got when the VNIR Band 1 and the raw SWIR Band 1 was used as the high and low resolution pan band respectively, and the optimal sharpened SWIR Band 2 image was got when the VNIR Band 7 and the downscaled 200 $\mathrm{m}$ VNIR Band 1 was used as the high and low resolution pan band respectively. For the image with fewer water areas and more vegetation such as the Area 1, the sharpened two SWIR band images from the first six VNIR bands used as the high resolution pan band were similar, by visual inspection, the sharpened SWIR images using the same VNIR band as the low resolution pan band were most similar to the raw SWIR Band images. The VNIR band with the higher CC value with the raw SWIR Band was selected, more texture information was injected the corresponding sharpened SWIR band image, and at that time another sharpened SWIR band image preserve the similar spectral and texture characteristics to the raw SWIR band image. In the future, a comprehensive evaluation over more study areas and with the different sharpening techniques and the reference images and assessment indices should be performed which will include comparison of the spectral and texture information of the classified objects.

\section{ACKNOWLEDGEMENTS}

This research work was jointly financially supported by the National Natural Science Foundation of China (Project No.41671422, 41661144030, 41471335), the Innovation Project of LREIS (Project No.088RA20CYA, 08R8A010YA), and National Science-Technology Support Plan Projects (Project No.2013BAD05B03). Thanks to China Manned Space Engineering for providing the Tiangong-II space science and application data products.

\section{REFERENCES}

Aiazzi, B., Alparone, L., Baronti, S., Santurri, L., and Selva, M., 2005. Spatial resolution enhancement of ASTER thermal bands, In: Proc. SPIE 5982, Image and Signal Processing for Remote Sensing XI, pp. 59821G. http://doi.org/10.1117/12.666545.

Du, Y., Zhang, Y. H., Ling, F., Wang, Q. M., Li W. B., and Li, X. D., 2016. Water Bodies' Mapping from Sentinel-2 Imagery with Modified Normalized Difference Water Index at 10-m Spatial Resolution Produced by Sharpening the SWIR Band. Remote Sensing, 8, 354. http://doi.org/10.3390/rs8040354.

Gao, L., Wang, Z. W., and Jiang, M., 2016. Assessment of fusion methods of ZY-3 satellite images. In: 2016 Academic Annual Conference of Jiangsu Provincial Society for Surveying, Mapping and Geoinformation, Nanjing, China, pp. 44-46. (In Chinese)

Matheson, D. S., and Dennison, P. E., 2012. Evaluating the effects of spatial resolution on hyperspectral fire detection and temperature retrieval. Remote Sensing of Environment, 124, pp. 780-792.

Nikolakopoulos, K. G., 2005. Comparison of six fusion techniques for SPOT 5 data. In: IEEE 2005 International
Geoscience and Remote Sesning Symposium, Seoul, Korea, Vol. 4, pp. 2811-2814.

Nikolakopoulos, K. G., 2006. Spatial resolution enhancement of EO-1 ALI bands, In: Proc. SPIE 6365, Image and Signal Processing for Remote Sensing XII, 63650A, Stockholm, Sweden. http://dx.doi.org/10.1117/12.688990.

Padwick, C., Deskevich, M., Pacifici, F., and Smallwood, S., 2010: WordView-2 Pan-Sharpening, In: ASPRS 2010 Annual Conference, San Diego, California, USA.

Pak, H., Choi, J., Park, N., and Choi, S., 2017. Sharpening the VNIR and SWIR Bands of Sentinel-2A Imagery through Modified Selected and Synthesized Band Schemes. Remote Sensing, 9, 1080. http://doi.org/10.3390/rs9101080.

Pour, A. B., and Hashim, M., 2013. Fusing ASTER, ALI and Hyperion data for enhanced mineral mapping. International Journal of Image and Data Fusion

http://dx.doi.org/10.1080/19479832.2012.753115

Sanchez-Ruiz, S., Piles, M., Sanchez, N., Martinez-Fernandez, J., Vall-llossera, M., Camps, A., 2014: Combining SMOS with visible and near, shortwave, thermal infrared satellite data for high resolution soil moisture estimates. Journal of Hydrology, 516, pp. 273-283.

Sarp, G., 2014. Spectral and spatial quality analysis of pan sharpening algorithms: A case study in Istanbul. European Journal of Remote Sensing, 47, pp. 19-28.

Young, D. K., 2015. Our SWIR Imagery product is now available, at the highest resolution ever from space, only from DigitalGlobe, Our eyes can play tricks on us but shortwave infrared (SWIR) imagery reveals all. http://blog.digitalglobe.com/technologies/our-eyes-can-playtricks-on-us-but-shortwave-infrared-swir-imagery-reveals-allpart-1-of-2/ (29/December/2017).

Vaiopoulos, A. D., and Karantzalos, K., 2016. Pansharpening on the narrow VNIR and SWIR spectral bands of Sentinel-2, In: The International Archives of the Photogrammetry, Remote Sensing and Spatial Information Sciences, Prague, Czech Republic, Vol. XLI-B7, pp. 723-730.

Vrabel, J., 1996. Multispectral Imagery Band Sharpening Study. Photogrammetric Engineering \& Remote Sensing, 62(9), pp. 1075-1083.

Vrabel, J. C., Doraiswamy, P., McMurtrey, J. E., and Stern, A., 2002. Demonstration of the accuracy of improved-resolution hyperspectral imagery, In: Proc. SPIE 4725, Algorithms and Technologies for Multispectral, Hyperspectral, and Ultraspectral Imagery VIII, Orlando, FL, USA. http://dx.doi.org/10.1117/12.478790.

Wahi, M., Taj-Eddine, K., and Laftouhi, N., 2013. ASTER VNIR \& SWIR Band Enhancement for Lithological Mapping A case study of the Azegour Area (Western High Atlas, Morocco). Journal of Environment and Earth Science, 3(12), pp. 33-44.

Wang, Q. M., Shi, W. Z., Li, Z. B., and Atkinson, P. M., 2016. Fusion of Sentinel-2 images. Remote Sensing of Environment, 187, pp. 241-252. 